\title{
EMG analysis of stereotyped voluntary movements in $\operatorname{man}^{1}$
}

\author{
MARK HALLETT, BHAGWAN T. SHAHANI, AND ROBERT R. YOUNG ${ }^{2}$ \\ From the Department of Neurology and the Laboratory of Clinical Neurophysiology, Harvard Medical School, \\ and Massachusetts General Hospital, Boston, Massachusetts, U.S.A.
}

SYNOPSIS EMG activity was recorded in biceps and triceps while subjects voluntarily flexed their elbows during a visual matching task. With fast flexion, the initial EMG was characterized by a triphasic pattern with a burst of activity first in biceps, then in triceps with a silent period in biceps, and finally in biceps again; these components were analysed quantitatively. Smooth flexion was characterized by continuous activity in biceps. Inhibition of tonic activity of triceps in relation to a fast flexion occurred in the $50 \mathrm{~ms}$ before the initiation of biceps activity. A patient with a severe pansensory neuropathy performed normally on these tasks. Physiological mechanisms underlying these patterns are analysed; an important conclusion is that the triphasic activity with fast flexion is 'centrally programmed'.

The precise timing of EMG activity in the several agonist and antagonist muscle groups of the human upper limb during voluntary projected movement was initially described in 1926 by Wachholder and Altenburger, and Wachholder (1928). They found different EMG patterns to be associated with movements of different velocity. With slow movements there was continuous EMG activity in the agonist, whereas the pattern with rapid movements consisted of a stereotyped alternation of activity between agonist and antagonist. Since then, there have been many contradictory reports suggesting, for example, that muscular activity in all movements is almost entirely restricted to the agonist (Wilkie, 1950), that the antagonist may be active during the entire movement (Barnett and Harding, 1955), or that the antagonist is active only at the end of a movement (Bierman and Ralston, 1965). Recent investigators studying EMG activity in projected movement have, in general, confirmed Wachholder and Altenburger's findings (Basmajian, 1967; Gottlieb et al., 1970; Marsden et al., 1972; Dijkstra and Denier van der Gon,

\footnotetext{
${ }^{1}$ Supported by the Parkinson's Disease Project of the Massachusetts General Hospital and the Allen P. and Josephine B. Green Foundation. 2 Address for reprints: Dr R. R. Young, Massachusetts General Hospital, Boston, Massachusetts 02114, U.S.A.

(Accepted 18 July 1975.)
}

1973; Hopf et al., 1973; Angel, 1974), but a complete and satisfactory understanding of the EMG patterns is still lacking. The present study is an attempt to define these patterns quantitatively for certain stereotyped movements in a normal population, to learn more about the physiological mechanisms involved, and to set standards by which to evaluate the performance of patients with various movement disorders.

\section{METHODS}

Eighteen normal subjects ( 15 males and three females ranging in age from 22 to 44 years) and one patient were studied. The latter is a $\mathbf{3 8}$ year old man with a severe pan-sensory neuropathy. He has little subjective appreciation of touch, pain, temperature, position, or vibration, has no deep tendon reflexes, and shows no silent periods in ongoing voluntary EMG activity to electrical stimulation of the mixed nerve. Details of his clinical state have been reported elsewhere (Adams et al., 1973); his limbs are considered essentially deafferented.

Studies were restricted to elbow flexion. Subjects sat facing an oscilloscope screen with their arm resting on a table with a Formica top that was covered with talcum powder to reduce friction. The arm was abducted approximately $90^{\circ}$ at the shoulder and the forearm was supinated so that biceps and brachialis become the prime flexors of the elbow 
(Basmajian and Latif, 1957). The initial elbow angle was $90^{\circ}$. EMG activity was recorded from the skin over the bellies of biceps and triceps using electrodes $3.0 \mathrm{~cm}$ apart. A lever strapped to the wrist extended vertically from a potentiometer which converted movement of the lower end of the lever into a variable voltage. The output was connected to the vertical axis of one channel of the dual beam oscilloscope observed by the subject. The vertical axis of the second channel of the oscilloscope was connected to a signal generator under the experimenter's control. The horizontal axis of both oscilloscope beams was set on rapid free-run so that the appearance on the screen was that of two horizontal lines.

Weights could be applied to the forearm by being connected to the lever by flexible wires strung over pulleys attached to the edge of the table. Weights were attached to the wires with light-weight electromagnets so that they could be released at any prescribed time. Release was signalled by the interruption of an electrical circuit between the electromagnet and the suspended weights. All this information was displayed on a second six-channel oscilloscope, where a permanent record was made with a Polaroid camera.

At the beginning of all experiments, subjects were asked to match the experimenter's line on the oscilloscope with their line. This was done by voluntarily adjusting the angle of their elbow and therefore the angle of the lever attached to their forearm and the potentiometer. At a time not predictable by the subject, the experimenter's line would suddenly drop to a new position which could be matched by means of elbow flexion. Subjects were told, 'Match the line as rapidly and as accurately as possible'. In practising this movement, subjects were continually urged to 'Try to do it faster'. The data were recorded only when the movement was well practised and appeared to be as rapid as possible. In most experiments, the movement consisted of approximately $10^{\circ}$ angular displacement of the elbow. A second set of instructions, usually presented after the first, asked the subjects to "Match the line smoothly, steadily, and accurately. You might have to go more slowly, but you should begin just as promptly'. In training for this task, subjects might be told, "That last movement was jerky; try to do it more smoothly'. Again, data were recorded only for practised movements.

Durations of the components of the EMG activity were measured with an accuracy of $\pm 10 \mathrm{~ms}$ from the photographic record and averaged for each subject. More attention was paid to the timing of the components than to their amplitudes which were merely approximated by measurement of the maximum voltage seen within any component.
The results using five different protocols are reported in this paper. The first was a fast flexion (FF) without any weights. In the second, designed to study antagonist inhibition (AI), the triceps was made to contract tonically by attaching a weight to the lever which, if unopposed, would act to flex the elbow. The subject was then told to match the line rapidly. The third was a smooth flexion (SF) without weights. The fourth protocol was arranged to get a passive extension (PE) of the elbow during a fast flexion effort. Approximately equal weights, usually about $2 \mathrm{~kg}$, were attached to both sides of the lever. With equal and opposite forces on the forearm there was no net pull and consequently no resting activity in either biceps or triceps. Subjects were instructed to match rapidly. Sometime between the displacement of the experimenter's line and the onset of biceps activity, the weight pulling in the direction of elbow flexion was electromagnetically released. This
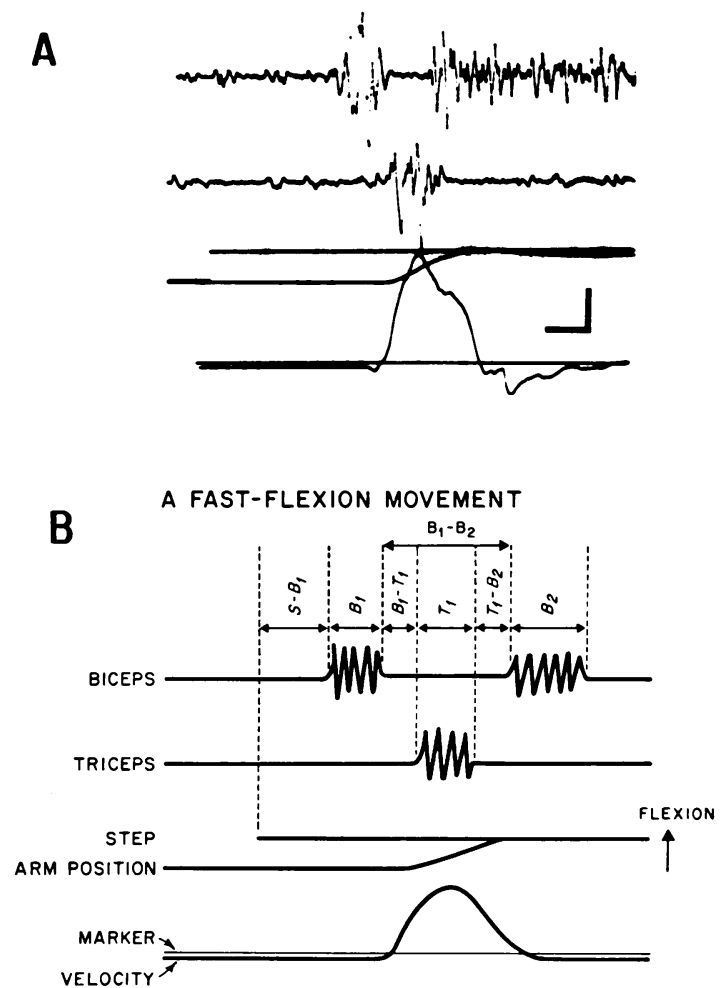

FIG. 1 Electromyographic activity in biceps and triceps during fast flexion of the elbow. A is a photographic record; the six oscillographic traces are diagrammatically described in B. Components of the EMG activity pictured in $\mathrm{B}$ are further defined in the text. Calibration: $50 \mathrm{~ms}, 500 \mu \mathrm{V}$. 
TABLE 1

NORMAL VALUES (MS) FOR FAST FLEXION EMG PARAMETERS*

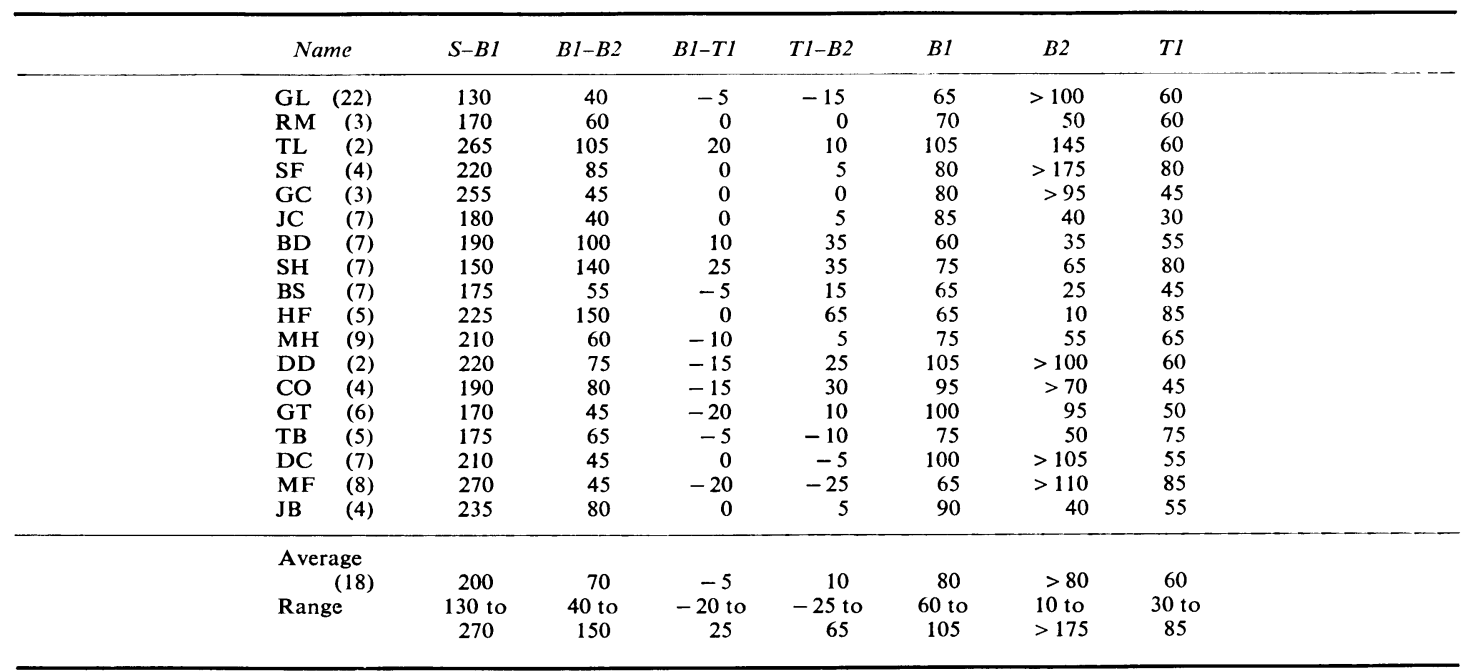

* Data are averages for the number of trials shown in parentheses after the subject's initials.

caused a passive extension of the elbow and tension on the triceps was reduced. When the biceps contracted, it was not successful in flexing the elbow or stretching the triceps. In performing this experiment the weight was dropped at random times in only onethird of the trials. In the fifth experiment (SU: smooth unload), the unloading reflex during smooth flexion was studied. A weight, usually about $2 \mathrm{~kg}$, was attached to the lever pulling in the direction of elbow extension, requiring tonic activity in the biceps to maintain elbow flexion of $90^{\circ}$. This weight was also released at random times in only one-third of the trials in which the subjects were asked to match smoothly.

\section{RESULTS}

FAST FLEXION (WITHOUT WEIGHTS) The initial experiment for most subjects was to flex the elbow trying to superimpose their line upon the experimenter's line as rapidly as possible (protocol FF). The initial part of the EMG pattern in all subjects could characteristically be described in the following manner (Fig. 1, A). There is a burst of action potentials in biceps (B1) followed by a period of silence in that muscle (B1-B2) during which triceps is active (T1). Triceps then becomes silent and biceps active again (B2). Usually further electrical activity is seen but is not analysed here because a pattern for the latter activity is much more difficult to discern than for the initial part.

In order to study the initial EMG patterno quantitatively we arbitrarily defined certain further parameters of the pattern which are illustrated in Fig. 1, B. B1, B1-B2, T1, and B2 refer to the durations of these components defined above. S-B1 refers to the time from the change in position of the experimenter's line to the beginning of B1 ('reaction time'). B1-T1 is defined as the interval between the end of $\mathrm{B} 1$ and the beginning of $\mathrm{T} 1$, and $\mathrm{T} 1-\mathrm{B} 2$ as the interval between the end of $\mathrm{T} 1$ and the beginning of B2; if there is overlap between B1 and $\mathrm{T} 1$, or $\mathrm{T} 1$ and $\mathrm{B} 2$, these intervals will have a negative value.

Analysis of the averaged data for 18 normal controls is shown in Table 1. The measurements of the parameters are moderately variable from trial to trial for a single subject and, as can be seen, there is a marked variability from one subject to another which gives rise to broad 'normal limits'. The parameters which seem least variable are B1, T1 and, to a lesser extent, B1-B2.

ANTAGONIST INHIBITION BEFORE AGONIST ACTIVA- 


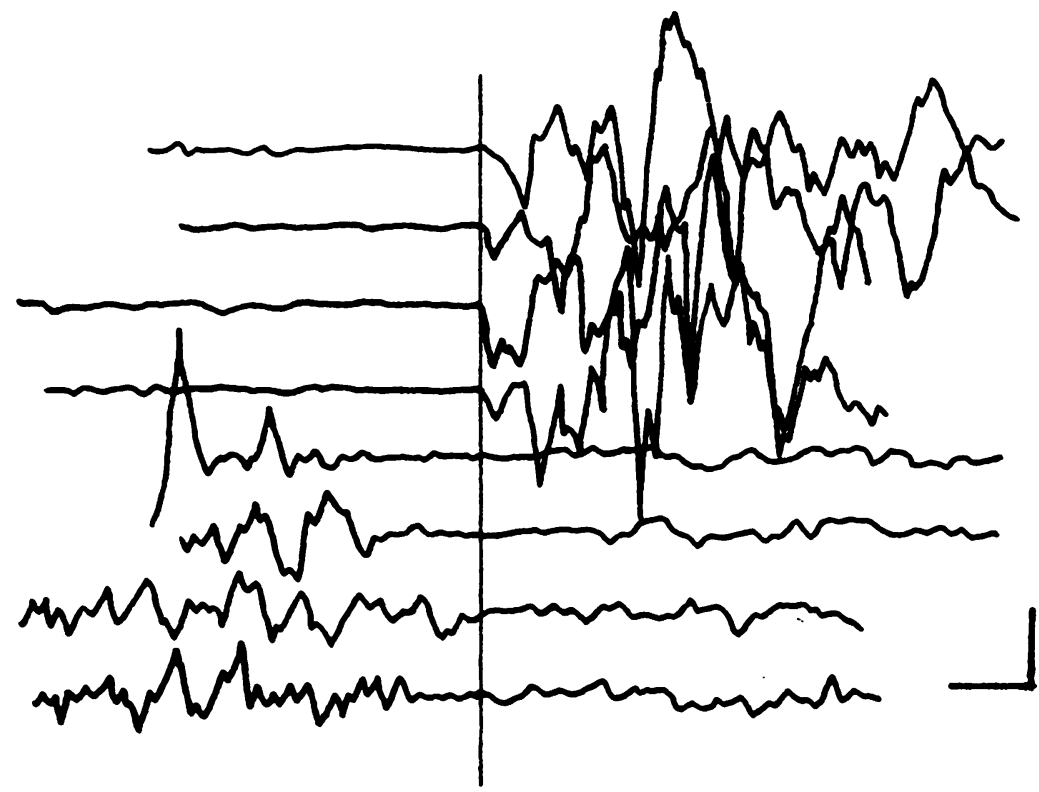

FIG. 2 Inhibition of tonic triceps activity before a fast elbow flexion. This is a composite of four individual records from one subject. The first line is a tracing of the biceps activity and the fifth line is a tracing of the triceps activity of the same trial. Lines 2 and 6, 3 and 7, and 4 and 8 are related similarly. The tracings are arranged so that the vertical line denotes the onset of B1. Calibration: $20 \mathrm{~ms}$, $200 \mu \mathrm{V}$.

TION In this experiment, the temporal relation between the cessation of EMG activity in a previously contracting antagonist and initiation of EMG activity in the agonist is investigated. The triceps is made to contract tonically before a rapid flexion movement (protocol AI).

Triceps activity ceased $0-50 \mathrm{~ms}$ before the initiation of $\mathrm{B} 1$. When triceps remained active up to the moment that biceps began, there was never any overlap of the activities in the two muscles. In addition to the cessation of triceps activity before the onset of B1, the amplitude of the terminal part of the triceps trace was often diminished. This phenomenon may best be thought of in a statistical sense-that is, the probability that EMG activity will be recorded from triceps becomes progressively less during the $50 \mathrm{~ms}$ before agonist activation (Fig. 2).
Four trials are recorded, the EMG traces from which are arranged so that the onset of B1 occurs at the time indicated by the vertical line. In this example, the triceps activity stopped 6 , 16,28 , and $48 \mathrm{~ms}$ before the initiation of $\mathrm{B} 1$.

SMOOTH FLEXION (WITHOUT WEIGHTS) Subjects were instructed to match the line smoothly (protocol SF). In this circumstance, completely different EMG patterns were seen. Most common was continuous activity in biceps without any triceps activity (Fig. 3). At other times, there was also essentially continuous and simultaneous triceps activity. Alternating biceps and triceps activity were not seen unless the movement was so quick as to mimic the fast flexion task.

PATTERNS IN 'DEAFFERENTED' MAN This subject

TABLE 2

VALUES (MS) FOR FAST FLEXION EMG PARAMETERS IN PATIENT WITH PAN-SENSORY NEUROPATHY

\begin{tabular}{|c|c|c|c|c|c|c|c|}
\hline Values & $S-B I$ & $B 1-B 2$ & $B 1-T 1$ & $T 1-B 2$ & $B 1$ & $B 2$ & $T I$ \\
\hline $\begin{array}{l}\text { Average } \\
\text { Range }\end{array}$ & $\begin{array}{c}90 \\
130- \\
350\end{array}$ & $\begin{array}{r}70 \\
20- \\
135\end{array}$ & $\begin{array}{r}-5 \\
-40- \\
30\end{array}$ & $\begin{array}{r}-10 \\
-135- \\
30\end{array}$ & $\begin{array}{l}85 \\
50- \\
130\end{array}$ & $\begin{array}{c}>105 \\
20- \\
410\end{array}$ & $\begin{array}{c}90 \\
20- \\
170\end{array}$ \\
\hline
\end{tabular}




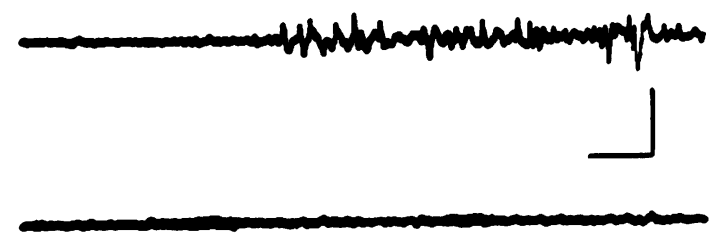

FIG. 3 EMG activity during a smooth flexion (SF). The first line is biceps activity, the second is triceps activity, the third is the step to be matched, and the fourth is the arm position. Calibration: $50 \mathrm{~ms}, 20 \mu \mathrm{V}$.
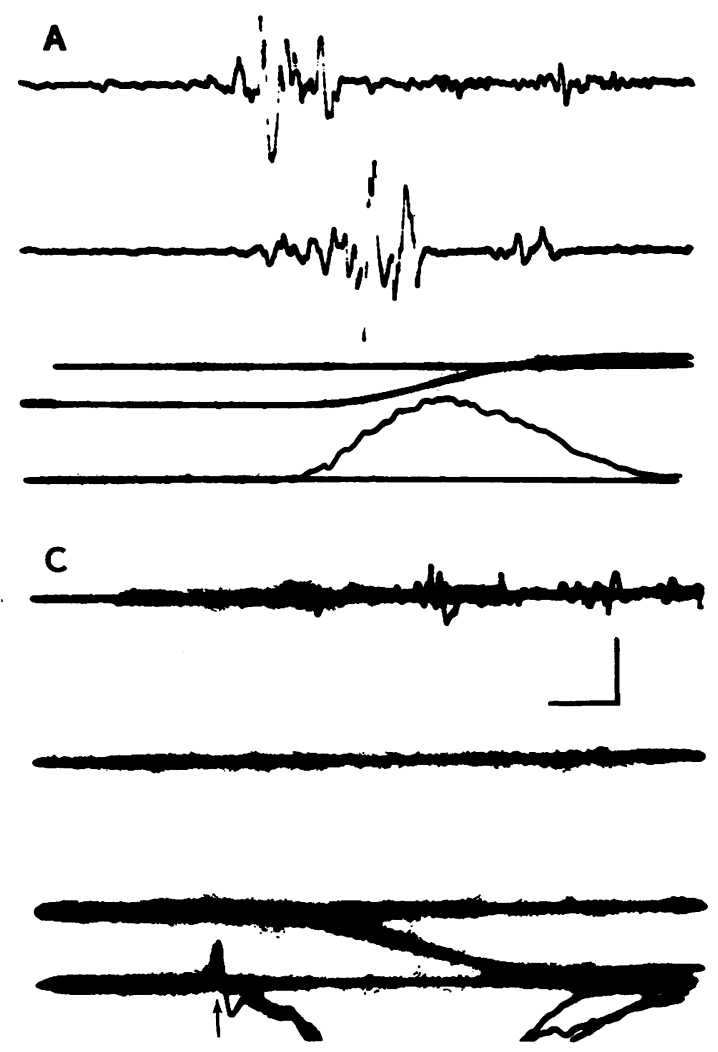

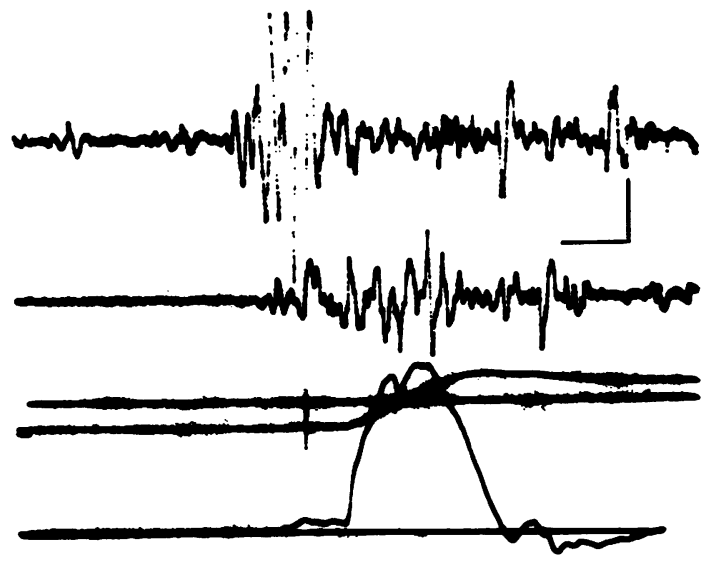

FIG. 4 EMG of a fast flexion movement (FF) for the patient with the pan-sensory neuropathy. Tracings are the same as in Fig. 1. Calibration: $50 \mathrm{~ms}, 200 \mu \mathrm{V}$.

FIG. 5 The effect of passive elbow extension on the fast flexion pattern (PE). The tracings in each part of the figure are similar to Fig. 1. A illustrates the EMG pattern with a $2 \mathrm{~kg}$ weight on each side of the arm. In $\mathrm{B}$, the weight pulling in the direction of elbow flexion is dropped at the time noted by the arrow. In $\mathrm{C}$, no voluntary movement is requested; the subject merely matches the baseline and the weight is dropped as in B. The record shows three superimposed trials. A small stretch reflex is seen in biceps, but triceps is quiet. Calibration: $50 \mathrm{~ms}, 500 \mu \mathrm{V}$. 


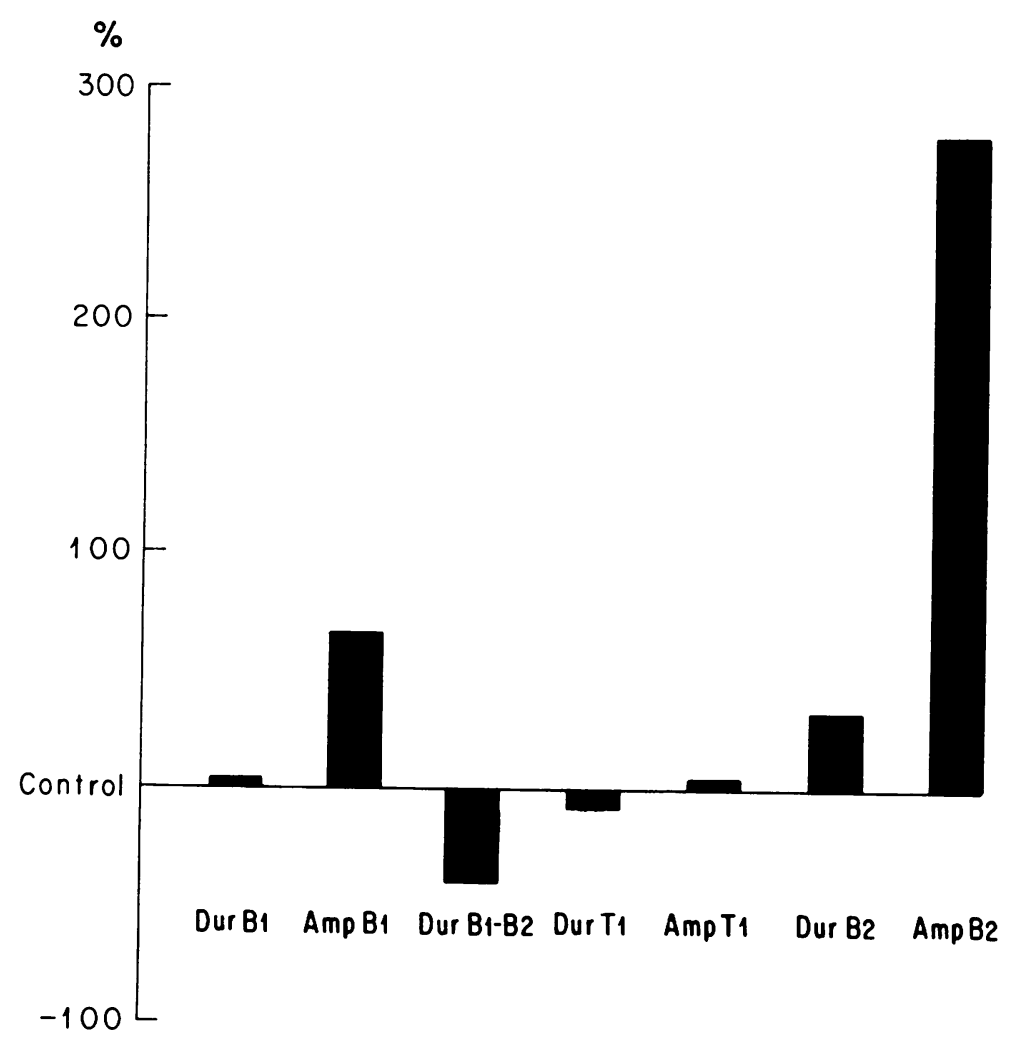

FIG. 6 Average changes in EMG components of the fast flexion pattern with passive extension. EMG components were measured and averaged for six subjects with and without passive elbow extension. The grand average for the percentage change of each component produced by release of the weight is plotted on the diagram.

was asked to do the three testing protocols described in the preceding sections. All three were performed normally. An example of the fast flexion (FF) pattern is shown in Fig. 4. Averages and ranges of the various parameters for 22 trials are shown in Table 2.

FAST FLEXION WITH PASSIVE EXTENSION The pattern during a fast flexion movement with both weights attached (Fig. 5) is similar to that seen without weights. When the weight pulling in the direction of elbow flexion is released, T1 was preserved both in duration and amplitude despite the fact that the arm was passively extended (Fig. 5, B). To show that this triceps activity is not merely part of a reflex pattern produced by the passive extension, the weight was released at times when the subject was not trying to make a voluntary movement and there is no triceps activity under this condition (Fig. 5, C).

Components of the EMG pattern were com- pared with and without release of the weight for six subjects. For B1, T1, and the amplitude of $\mathrm{T} 1$ there was no significant change with release of the weight, some subjects showing a small increase and some a small decrease. B1-B2 increased in four subjects, remained the same for one, and decreased slightly for the last. The amplitude of B1 increased slightly for all subjects and the amplitude of B2 increased to a much larger extent in all subjects. The average percentage change in these parameters produced by release of the weight is illustrated in Fig. 6 .

UNLOADING REFLEX DURING SMOOTH FLEXION Unloading responses were obtained during a smooth flexion (protocol SU). On control trials when the weight was not dropped, the rate and amplitude of the biceps EMG increased after a certain reaction time. On the experimental trials, the weight was dropped at various times after (and occasionally even before) four subjects were given the stimulus to move. At no time for 
any subject did the unloading reflex change significantly in its duration.

\section{DISCUSSION}

NORMAL EMG PATTERNS ASSOCIATED WITH A FAST MOVEMENT In order to understand how the nervous system controls movement, the details of the movement itself must be understood. Even the specific EMG patterns recorded from agonist and antagonist muscles during a simple rapid flexion movement are rather complex. At the beginning of the movement any activity in antagonist groups ceases and the agonist activity appears in two bursts separated by a silent period; activity in the antagonist is noted, to a close approximation, during this silent period. The physiological question is, can these reproducible patterns be explained entirely as a product of segmental reflex interactions? If not, it would be necessary to assume the presence of suprasegmental 'centrally programmed' input directly to the motor neurone pools. Answers can be obtained by looking at the interactions of H-reflexes, tendon reflexes, muscle stretching or unloading during the course of the movement.

EMG activity in a tonically active antagonist muscle ceases before agonist activation and thus, in the experimental setting, $\mathrm{AI}$ is an earlier sign of voluntary movement than the onset of agonist activity itself. This phenomenon has been demonstrated in other settings, both in man (Hufschmidt and Hufschmidt, 1954; Gottlieb et al., 1970) and in monkeys (Evarts, 1974). Because this phenomenon is present in the patient with the pan-sensory neuropathy, we suggest that it can be accounted for by a central signal directly onto the alpha motor neurone pool. Other studies, with unloading (Angel et al., 1970) and H-reflexes (Pierrot-Deseilligny et al., 1971; Simoyama and Tanaka, 1974), have suggested that there is also normally a decrease in the influence of the gamma loop at this same time.

The presence of the double burst pattern of the agonist in the deafferented patient suggests that it is also centrally programmed. The silent period between the bursts must come from an inhibitory, or decrease of excitatory, influence of this suprasegmental signal. Garland and Angel (1971) showed that B1 is not affected by unload- ing, while B2 is. Using passive muscle stretch rather than unloading (the PE task), our results show a slight increase in amplitude of $\mathrm{B} 1$, a mild foreshortening of B1-B2, and a large increase in the amplitude and slight increase in duration of B2. Thus, segmental afferent input has little effect on the agonist early in the fast flexion movement, but increases its influence as the movement progresses. On the other hand, the amplitude of the H-reflex of the agonist increases before, and reaches a peak about, the time of the onset of $\mathrm{B} 1$; it then gradually declines throughout the course of the movement (Gottlieb et al., 1970; Pierrot-Deseilligny et al., 1971). It is of interest that polysynaptic reflexes in man also have two components (Shahani and Young, 1971, 1972) which appear to have similar characteristics to those seen here for voluntary movements, but the physiological interpretation of this similarity is uncertain.

Most investigators (Basmajian, 1967; Gottlieb et al., 1970) assume that $\mathrm{T} 1$ is a result of a stretch reflex produced by passive extension of triceps by B1. Two results here disprove that $\stackrel{\circ}{\circ}$ 응 hypothesis. $T 1$ is present in the deafferented $\stackrel{\mathbb{Q}}{2}$ patient; and $\mathrm{T} 1$ is present, and to a large extent 0 unmodified, even when the elbow is passively 8 $ᄋ$ extended (and triceps NOT stretched) during an $\frac{0}{3}$ attempt at fast flexion. It appears, therefore, that $\mathrm{T} 1$, like $\mathrm{B} 1$, is part of a centrally pre-pro- $\stackrel{\vec{\varphi}}{\vec{\varphi}}$ grammed pattern which is not particularly subject to segmental influence. Because $\mathrm{T} 1$ is not exactly co-extensive with B1-B2, and because T1 is not subject to segmental influence, while $\mathrm{B} 1-\mathrm{B} 2$ is, $\mathrm{T} 1$ and $\mathrm{B} 1-\mathrm{B} 2$ are apparently organized separately and not merely reciprocal. The amplitude of the $\mathrm{H}$-reflex in the antagonist is reduced during the course of the movement (Gottlieb et al., 1970), but this reduction is transiently less marked about the time of T1.

For both the agonist and antagonist, there are at least three, not necessarily independent, controlling forces which differ in time course. First is the pre-programmed suprasegmental signal directly to the alpha motor neurone pool. Second is a similar signal directed to the gamma motor neurone pool, which influences the alpha motor neurones indirectly via the servo-loop. Third is a signal thought, on the basis of $\mathrm{H}$ reflex studies, to control Ia feedback gain (Gottlieb et al., 1970). The time course of these 


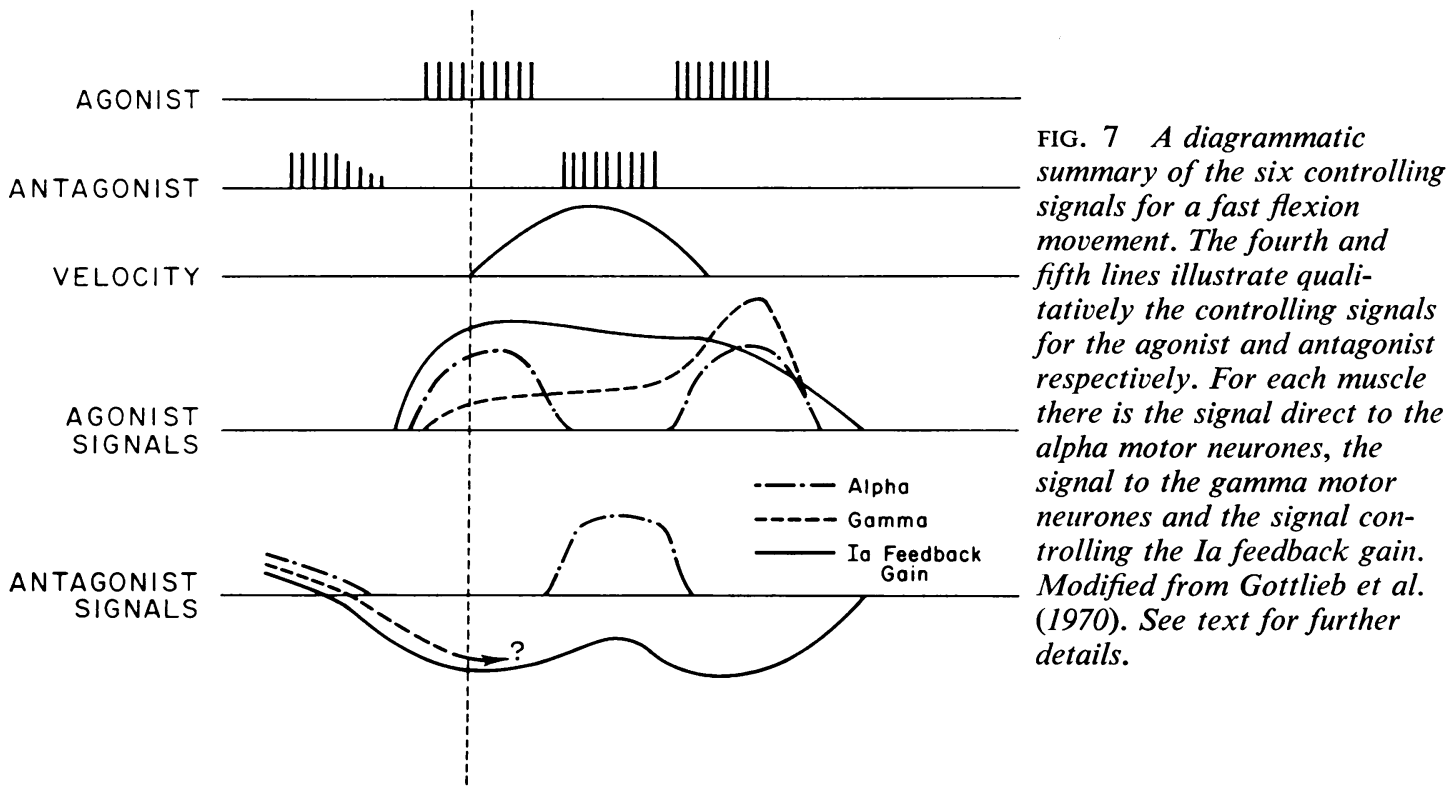

signals, so far as is known, is diagrammatically represented in Fig. 7, which is modified from Gottlieb et al. (1970).

Our conclusion is that the initial 'triphasic' pattern of fast flexion is centrally preprogrammed; $\mathrm{B} 1$ and $\mathrm{T} 1$ are relatively immune to perturbation by peripheral influences, but B2 is at least partially modifiable. Stetson and McGill (1923) had suggested that ballistic movements are too rapid for feedback to have any influence-that is, 'preprogrammed'. An analysis of the velocity of these movements also suggested preprogramming (Bouisset and Lestienne, 1974). Hopf et al. (1973) have come to the same conclusion as we have on the basis of interaction of rapid movements with vibration, direct electrical stimulation, and stretches of muscle.

The normal ranges of duration of certain features-B1, T1, and 'inhibition' of antagonist activity before B1-B2-are sufficiently narrow for them to be useful as standards for evaluation of patients with movement disorders. It should be noted that the values reported here are similar to previously reported approximate values for similar movements (Dijkstra and Denier van der Gon, 1973; Hopf et al., 1973; Hufschmidt and Hufschmidt, 1954; Hufschmidt, 1954). The ranges of normal values for the other parameters of the fast flexion movement are much broader. For example, the range of values for duration of $\mathrm{B} 2$ is so extensive that it would be difficult to specify an abnormal performance. One might guess that its variability arises from the strong influence of feedback on this component and the subject's appraisal of whether he is over- or under-shooting the mark.

NORMAL EMG PATTERNS ASSOCIATED WITH A SMOOTH FLEXION MOVEMENT Evidence is mounting that the central nervous mechanisms controlling rapid, ballistic movements are separate from those responsible for slower, smooth 'ramp movements' (Kornhuber, 1971; DeLong and Strick, 1974). It seemed useful, then, to try to establish standards of performance for this latter kind of movement as well. In all subjects, there was continuous activity of the agonist and in no subject was there a triphasic pattern as noted above. Thus, it is possible electromyographically to distinguish, in this paradigm, ballistic from ramp movements.

It had already been observed by Marsden et al. (1972) that unloading a muscle during a steady movement produces a momentary silence in the ongoing EMG, an 'isotonic' unloading reflex similar to the more traditional 'isometric' 
unloading reflex. In addition to confirming that, we have made further observations during both the period between the stimulus and response and the first part of the response itself. A similar brief period of EMG silence was seen in both instances. In this respect, fast movements and smooth movements are different, since segmental input does not influence the first part of the fast flexion pattern. Thus, in addition to the EMG differences, the controlling signals for fast and smooth movements appear to be different. For smooth movements, the servo-loop seems to be continuously active and strongly influential from the beginning of the movement.

\section{REFERENCES}

Adams, R. D., Shahani, B. T., and Young, R. R. (1973). A severe pansensory familial neuropathy. Transactions of the American Neurological Association, 98, 13-15.

Angel, R. W. (1974). Electromyography during voluntary movement: The two-burst pattern. Electroencephalography and Clinical Neurophysiology, 36, 493-498.

Angel, R. W., Garland, H., and Alston, W. (1970). Interaction of spinal and supraspinal mechanisms during voluntary innervation of human muscle. Experimental Neurology, 28, 230-242.

Barnett, C. H., and Harding, D. (1955). The activity of antagonist muscles during voluntary movement. Annals of Physical Medicine, 2, 290-293.

Basmajian, J. V. (1967). Muscles Alive. Their Functions Revealed by Electromyography, 2nd edn. Williams and Wilkins: Baltimore.

Basmajian, J. V., and Latif, A. (1957). Integrated actions and functions of the chief flexors of the elbow: a detailed electromyographic analysis. Journal of Bone and Joint Surgery, 39-A, 1106-1118.

Bierman, W., and Ralston, H. J. (1965). Electromyographic study during passive and active flexion and extension of the knee of the normal human subject. Archives of Physical Medicine and Rehabilitation, 46, 71-75.

Bouisset, S., and Lestienne, F. (1974). The organization of a simple voluntary movement as analysed from its kinematic properties. Brain Research, 71, 451-457.

DeLong, M. R., and Strick, P. L. (1974). Relation of basal ganglia, cerebellum, and motor cortex units to ramp and ballistic limb movements. Brain Research, 71, 327-335.

Dijkstra, S. J., and Denier van der Gon, J. J. (1973). An analog computer study of fast, isolated movements. Kybernetik, 12, 102-110.
Evarts, E. V. (1974). Sensorimotor cortex activity associated with movements triggered by visual as compared to somesthetic inputs. In The Neurosciences. Third Study Program, pp. 327-337. Edited by F. O. Schmitt and F. G. Worden. MIT Press: Cambridge, Mass.

Garland, H., and Angel, R. W. (1971). Spinal and supraspinal factors in voluntary movement. Experimental Neurology, 33, 343-350.

Gottlieb, G. L., Agarwal, G. C., and Stark, L. (1970). Interactions between voluntary and postural mechanisms of the human motor system. Journal of Neurophysiology, 33, 365-381.

Hopf, H. C., Lowitzsch, K., and Schlegel, H. J. (1973). Central versus proprioceptive influences in brisk voluntary movements. In New Developments in Electromyography and Clinical Neurophysiology, vol. 3, pp. 273-276. Edited by J. E. Desmedt. Karger: Basel.

Hufschmidt, H. J. (1954). Die rasche Willkürkontraktion. Beitrag zur elektromyographichen Analyse der menschlichen Willkürmotorik. Zeitschrift für Biologie, 107, 1-24.

Hufschmidt, H. J., and Hufschmidt, T. (1954). Antagonist inhibition as the earliest sign of a sensory-motor reaction. Nature, 174, 607.

Kornhuber, H. H. (1971). Motor functions of cerebellum and basal ganglia: The cerebello-cortical saccadic (ballistic) clock, the cerebellonuclear hold regulator, and the basal ganglia ramp (voluntary speed smooth movement) generator. Kybernetik, 8, 157-162.

Marsden, C. D., Merton, P. A., and Morton, H. B. (1972). Servo action in human voluntary movement. Nature, $\mathbf{2 3 8}$, 140-143.

Pierrot-Deseilligny, E., Lacert, P., and Cathala, H. P. (1971). Amplitude et variabilité des réflexes monosynaptiques avant un mouvement volontaire. Physiology and Behavior, 7, 495-508.

Shahani, B. T., and Young, R. R. (1971). Human flexor reflexes. Journal of Neurology, Neurosurgery, and Psychiatry, 34, 616-627.

Shahani, B. T., and Young, R. R. (1972). Human orbicularis oculi reflexes. Neurology (Minneap.), 22, 149-154.

Simoyama, M., and Tanaka, R. (1974). Reciprocal 1a inhibition at the onset of voluntary movements in man. Brain Research, 82, 334-337.

Stetson, R. H., and McGill, J. A. (1923). Mechanisms of the different types of movements. Psychological Monographs, 32, 18-45.

Wachholder, K. (1928). Willkürliche Haltunghund Bewegung insbesondere im Lichte electrophysiologischer Untersuchungen. Ergebnisse der Physiologie, 26, 568-775.

Wachholder, K., and Altenburger, H. (1926). Beiträge zur Physiologie der willkürlichen Bewegung. 10. Einzelbewegungen. Pflügers Archiv für die gesamte Physiologie des Menschen und der Tiere, 214, 642-661.

Wilkie, D. R. (1950). The relation between force and velocity in human muscle. Journal of Physiology, 110, 249-280. 